\title{
Nuclear Lamins in Cell Regulation and Disease
}

\author{
T. Shimi, V. Butin-IsRaeli, S.A. AdAm, AND R.D. Goldman \\ Department of Cell and Molecular Biology, Northwestern University Feinberg School of Medicine, \\ Chicago, Illinois 60611 \\ Correspondence: r-goldman@northwestern.edu
}

\begin{abstract}
The nuclear lamins are type $\mathrm{V}$ intermediate filament proteins that form meshworks at the inner aspect of the nuclear envelope and are also present throughout the nuclear interior. Through these meshwork structures, lamins regulate the shape, size, and mechanical properties of the nucleus. During the last 25 years, the Goldman laboratory has studied the organization and dynamic properties of the lamins in the nucleus. These studies have characterized the role of lamin phosphorylation in nuclear lamina assembly and disassembly during mitosis. Furthermore, our studies have demonstrated a role for the lamins in chromatin modification and epigenetics, transcription, and DNA replication. Recently, the discovery of numerous mutations in the gene encoding A-type lamins causing the collection of diseases known as laminopathies has provided new insights into the roles of lamins in cellular regulation and differentiation.
\end{abstract}

The nuclear lamins were originally described as the major proteins of the nuclear lamina located in the periphery of the nucleus (Fawcett 1966; Patrizi and Poger 1967; Aaronson and Blobel 1975). Our interest in nuclear lamins began 25 years ago when several laboratories, including our own, discovered that the lamins are intermediate filament (IF) proteins. Subsequently, the lamins were designated as the type $\mathrm{V}$ members of the IF protein family. Here we review the work from our own laboratory on these nuclear proteins, now considered to be major building blocks of nuclear architecture and chromatin organization.

\section{NUCLEAR LAMINS ARE NUCLEOSKELETAL INTERMEDIATE FILAMENT PROTEINS}

Nuclear lamins are the major components of the nuclear lamina (Fig. 1). The lamina provides a molecular interface that anchors both the nuclear membrane-embedded nuclear pore complexes and peripheral elements of chromatin within the nuclear envelope (NE) (Fig. 2). In the mid 1970s, lamins were first isolated along with nuclear pore complexes by detergent and salt fractionation of rat liver nuclei (Aaronson and Blobel 1975; Gerace et al. 1978). Subsequently, we found that the nuclear lamins cofractionated with cytoskeletal IF preparations. Later, the lamins were classified as type V IF proteins based on their biochemical properties and amino acid sequences (Zackroff et al. 1984; Goldman et al. 1986; McKeon et al. 1986). Subsequently, the lamins were subdivided into Atype lamins (lamins A and C) and B-type lamins (lamins B1 and B2) (Dechat et al. 2008). Lamins A and C are derived from a single gene ( $L M N A)$ by alternative splicing and are expressed primarily in differentiated cells. Lamins $\mathrm{B} 1$ and $\mathrm{B} 2$ are encoded by $L M N B 1$ and $L M N B 2$, respectively, and at least one of these isoforms is expressed in all cells throughout development in vertebrates (Dechat et al. 2008).

\section{BIOCHEMICAL PROPERTIES OF NUCLEAR LAMINS}

Similar to all cytoskeletal IF proteins, lamins consist of an $\alpha$-helical central rod domain flanked by short globular amino-terminal "head" and longer carboxy-terminal "tail" domains. However, lamins are distinguished from the cytoskeletal members of the IF protein family by several unique structural features. First, the lamin carboxy-terminal tail contains a highly conserved immunoglobulin fold (Ig-fold), a structural motif involved in protein-protein and protein-nucleic acid interactions. The tail also contains a nuclear localization signal (NLS) required for transport of the nuclear lamins into the nucleus and their assembly into the lamina (Adam et al. 2008; Dechat et al. 2008; Shumaker et al. 2008). Finally, vertebrate lamins have an additional 6-heptad repeat in their central rod domain not found in nonvertebrate lamins or in cytoskeletal IF proteins. The basic building blocks of lamin polymers are coiled-coil dimers formed by the central rod domain that can polymerize into a variety of higher-order structures. The head and tail regions of lamins are also involved in lamin polymerization as is evident from experiments with lamin deletion mutants (Goldman et al. 1992; LopezSoler et al. 2001; Shumaker et al. 2005). In vitro, cytoplasmic IF proteins form 10-nm filaments; however, the end point for lamin polymerization is the formation of highly ordered paracrystalline arrays (Zackroff et al. 1984; Aebi et al. 1986; Goldman et al. 1986; Parry et al. 1987). The importance of the central rod in the assembly and structure of the lamins has been emphasized by the finding that lamin A with a point mutation in this domain, known to cause an atypical form of the premature aging disease progeria, forms structurally defective paracrystals (Fig. 3) (Taimen et al. 2009). We have also studied the steps involved in the assembly of lamins into the lamina in situ. Within minutes after microinjecting pre-lamin A into the cytoplasm (see below), the protein is found in foci 

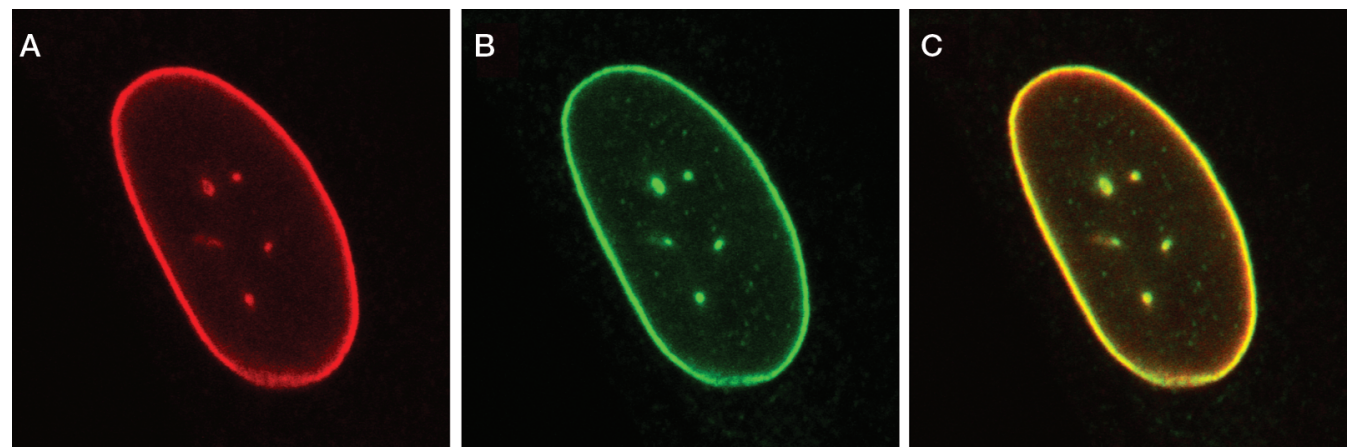

Figure 1. Nuclear lamins in normal human diploid fibroblast cells. The nuclear lamins were detected with specific antibodies to lamins $\mathrm{A}$ and $\mathrm{C}(A)$ or lamin B2 $(B)$. (C) Overlay of the two channels, showing that A-type and B-type lamin networks colocalize. Note also the localization of both A-type and B-type lamins to a nucleoplasmic "veil."

distributed throughout the nucleoplasm. After $6 \mathrm{~h}$, the foci decrease in size and number as the microinjected lamin A becomes associated with the endogenous nuclear lamina (Goldman et al. 1992). However, the relationships between the steps in assembly and the posttranslational modifications involved in the conversion of pre-lamin A to mature lamin A (see below) remain unknown.

\section{ORGANIZATION OF NUCLEAR LAMINS}

Nuclear lamins have been reported to form filamentous structures within the lamina of some cell types. In the middle 1980s, germinal vesicle NEs of Xenopus laevis oocytes were found to contain a regular meshwork of lamin filaments ranging from $\sim 10$ to $15 \mathrm{~nm}$ as observed by electron microscopy (Aebi et al. 1986). More recently, we have described a meshwork organization of lamins in the NE of mammalian cells by high-resolution light microscopy (Shimi et al. 2008). These lamina meshworks are composed of separate but overlapping arrays of A-type and B-type lamin fibers. There is also evidence that the A-type and B-type lamin structures interact directly with each other. This is based on fluorescence resonance energy transfer (FRET) experiments showing interactions between lamins A and B1 within the lamina of living cells (Moir et al. 2000b; Delbarre et al. 2006). The expression of lamin B1 appears to be critical for forming both A-type and B-type lamin meshworks (Shimi et al. 2008). For example, when lamin B1 expression is silenced in HeLa cells, the mesh size of the lamin A/C and B2 meshworks is significantly increased. Lamin B1 silenced cells also frequently develop nuclear blebs with an extensively enlarged lamin A/C meshwork, devoid of lamin B2 and NPCs (Fig. 4). Many of these structural changes in lamin meshworks are also found to various degrees in cells expressing lamin $\mathrm{A} / \mathrm{C}$ point mutations or deletions known to cause a wide spectrum of human diseases (Fig. 5) (Goldman et al. 2004; Kandert et al. 2007; Dechat et al. 2008; Taimen et al. 2009).

The dynamic properties of lamins in the lamina and also in the nucleoplasm have been studied mainly by mobility measurements of green fluorescent protein (GFP)-fused lamins in living cells (Moir et al. 2000b; Shimi et al. 2008). Fluorescence recovery after photobleaching (FRAP) and fluorescence correlation spectroscopy (FCS) have revealed that both A-type and B-type lamins exchange subunits slowly within the lamina throughout interphase except during the $G_{1}$ phase of the cell cycle, a period of active increases in nuclear size (Moir et al. 2000b; Shimi et al. 2008). The A-type and B-type lamins are also present throughout the nucleoplasm, albeit at lesser concentrations than found in the lamina (Goldman et al. 1992; Moir et al. 2000b; Shimi et al. 2008). The Btype lamins located in the nucleoplasm are relatively static, similar to those in the lamina, whereas the nucleoplasmic A-type lamins are highly dynamic, as determined by fluorescence correlation spectroscopy. Silencing Btype lamin expression dramatically increases the mobility of nucleoplasmic lamin A, showing that there are also interactions between the A-type and B-type lamins through-

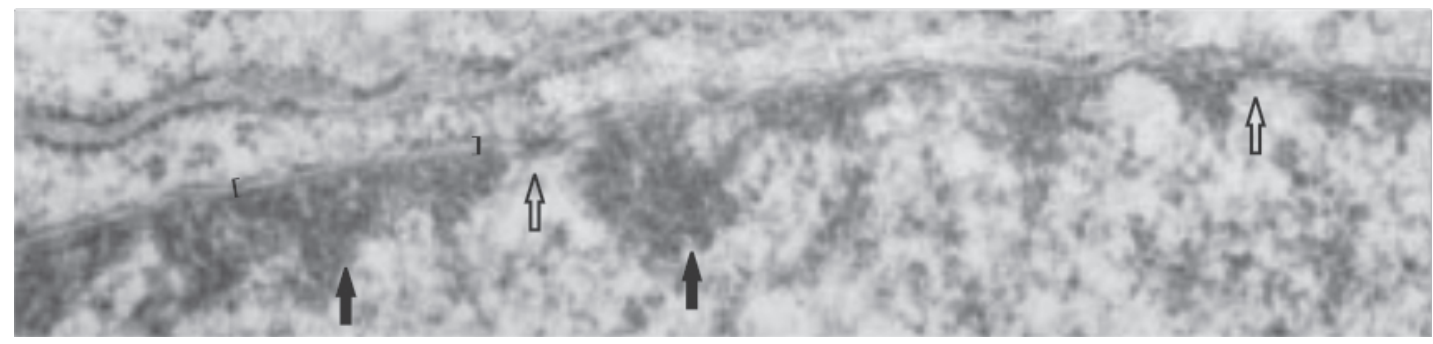

Figure 2. Segment of the nuclear envelope viewed by negative stain transmission electron microscopy. Nuclear envelope is comprised of a double-membrane bilayer (brackets), interrupted by nuclear pore complexes (open arrows). Peripheral heterochromatin can be seen closely apposed to the inner side of the nuclear membrane (closed arrows). Although the thin nuclear lamina is not visible in this image, lamin meshworks lie between the inner nuclear membrane and peripheral heterochromatin. (Adapted from Goldman et al. 2004.) 

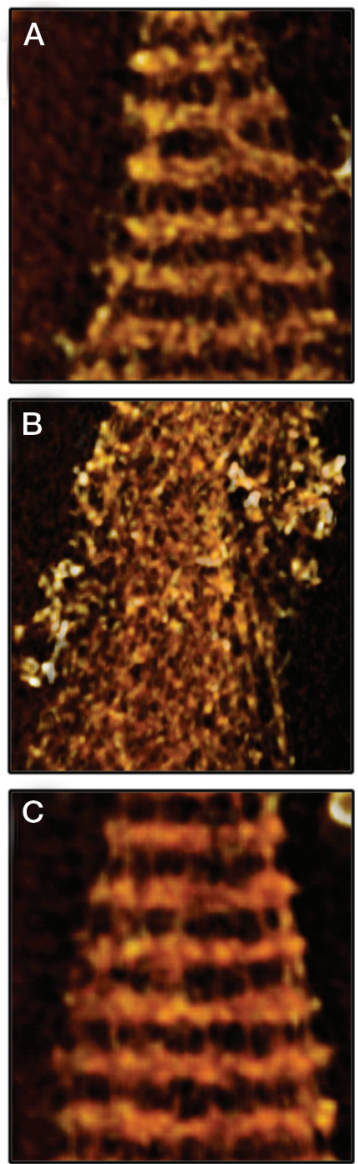

Figure 3. Cryo-electron tomography (cryo-ET) analysis of paracrystals assembled from WT-LA $(A)$, E145K-LA $(B)$, and LA $\Delta 50 /$ progerin $(C)$. Bacterially expressed and purified WT-LA, E145K-LA, and LA $\Delta 50 /$ progerin were allowed to self-assemble into paracrystals in vitro and analyzed by cryo-ET. Pseudocolored higher-magnification images show volume-rendering views of $30-\mathrm{nm}$-thick tomographic slices. Both WT-LA and LA $\Delta 50$ /progerin paracrystals show similar repeat patterns, in contrast to E145K-LA paracrystals, which are significantly less well ordered. (Adapted from Taimen et al. 2009.)

out the nucleoplasm (Fig. 6) (Shimi et al. 2008). These differences in the dynamic behavior of nucleoplasmic Atype and B-type lamins are supported by their biochemical extraction properties. Nucleoplasmic lamin A can be extracted in detergent/high-ionic-strength solutions, but nucleoplasmic lamin B1 is insoluble under these conditions (Moir et al. 2000b).

\section{LAMIN MESHWORK DISASSEMBLY DURING NUCLEAR ENVELOPE BREAKDOWN}

During the process of nuclear envelope breakdown (NEBD) at the onset of mitosis or meiosis, the polymerized lamins within the lamina meshworks are disassembled. Our studies of the role of the lamins in this process have involved the use of the germinal vesicles of the surf clam, Spisula solidissima, oocytes. During NEBD in clam oocytes, the nuclear lamina undergoes a marked reduction in size accompanied by the extensive phosphorylation of
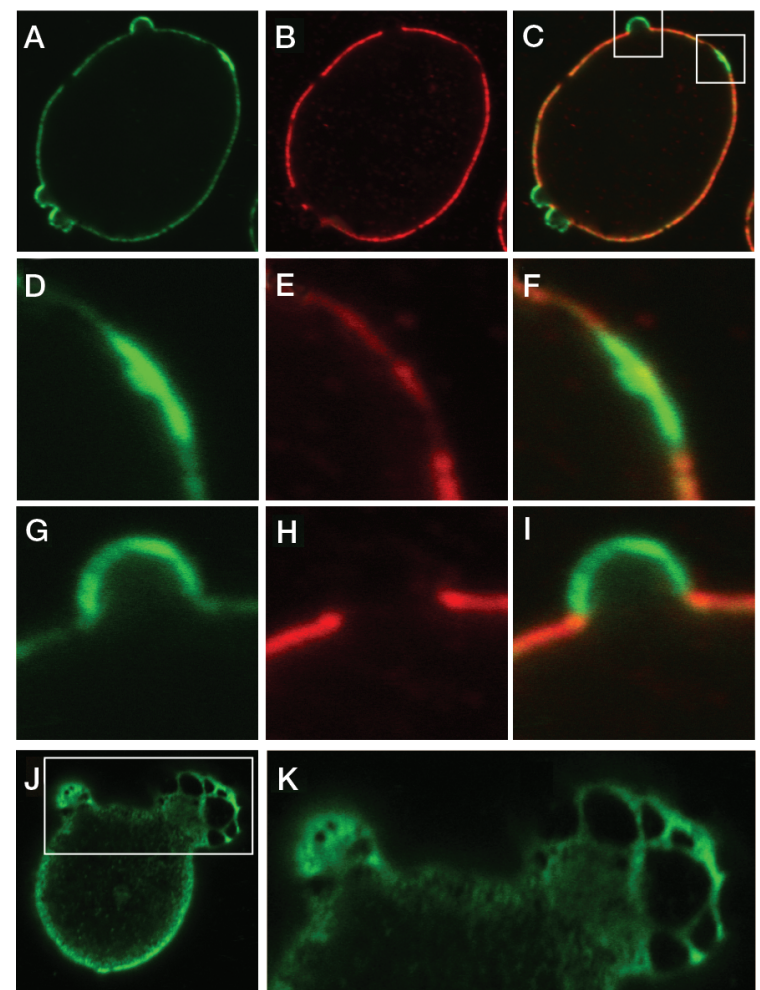

Figure 4. Lamins A, C, and B2 were colocalized in lamin B1 silenced cells. Immunostaining for lamins A and $\mathrm{C}(A)$ and B2 $(B)$ and overlay of the two images $(C)$ in the nucleus of a cell where lamin B1 has been silenced show the separation of A-type and B-type lamin networks. $(D-I)$ Magnifications of areas outlined by boxes in $C$. $(J, K)$ Surface view of another nucleus of a lamin $\mathrm{B} 1$ silenced cell containing two blebs. The larger of these blebs has a significantly enlarged lamin $\mathrm{A} / \mathrm{C}$ meshwork; see the higher-magnification view of the rectangular area in $K$, which is enlarged $\sim 2.4$-fold. (Modified, with permission, from Shimi et al. 2008.)

the single type of lamin present in the clam (Dessev et al. $1988,1989,1990,1991)$. As the clam lamin is phosphorylated, it is released in a soluble form from the lamin polymers (Dessev and Goldman 1988; Dessev et al. 1989, 1990, 1991). In the soluble fraction, lamin monomers and dimers are found to be phosphorylated at sites adjacent to the amino-terminal and carboxy-terminal ends of the rod domain by the complex consisting of the kinase CDK1 and cyclin B (Peter et al. 1990).

Similarly, disassembly of the nuclei of vertebrate somatic cells expressing both A-type and B-type lamins is regulated by CDK1 phosphorylation (Heald and McKeon 1990). The A-type and B-type lamins disassemble in sequential order with the A-type lamins dissociating from the lamina first, followed by the disassembly and fragmentation of the B-type lamins during the prophase/ prometaphase transition (Georgatos et al. 1997). FRAP and fluorescence loss in photobleaching (FLIP) have revealed that A-type lamins are freely diffusible throughout 

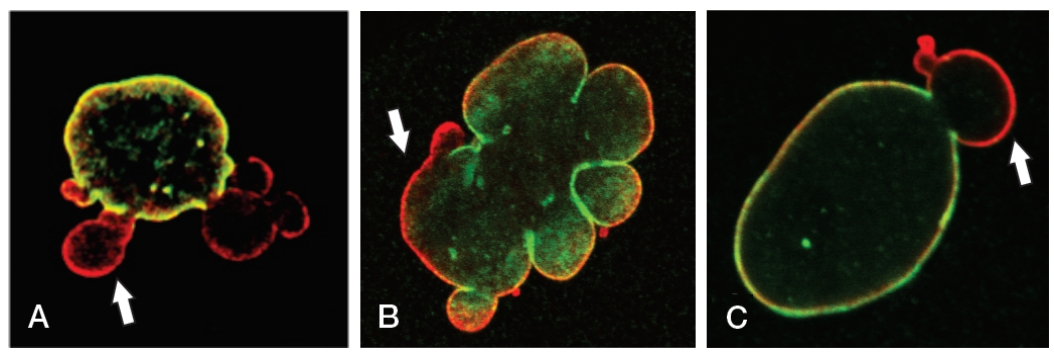

Figure 5. Structural changes in lamin meshworks in cells expressing different lamin A/C point mutations. Dermal fibroblasts from three progeria patients were fixed and immunostained to localize lamins $\mathrm{A}$ and $\mathrm{C}$ (red) and $\mathrm{B} 2$ (green). Each cell line expresses a different mutant lamin A or lamins A and C: progerin $(A), \mathrm{E} 145 \mathrm{~K}(B)$, or $\mathrm{S} 143 \mathrm{~F}$ $(C)$. The arrows indicate lamin- $\mathrm{A} / \mathrm{C}$ rich, lamin-B-deficient blebs.

the cell at metaphase (Moir et al. 2000b; Dechat et al. 2007), whereas fragments of the B-type lamin network remain associated with the nuclear membranes dispersed throughout mitotic cells (Georgatos et al. 1997). In mitotic spindles assembled in vitro in Xenopus egg extracts, the major B-type lamin, lamin B3, assembles into the spindle matrix during mitosis in a Ran-, Nudel-, and dynein-dependent manner (Tsai et al. 2006; Ma et al. 2009). Depletion of lamin B3 in this system, as well as the addition of dominant negative lamin B3 mutants, results in defects in spindle assembly (Tsai et al. 2006).

\section{REASSEMBLY OF LAMINS DURING THE FORMATION OF NUCLEI IN DAUGHTER CELLS}

At the end of mitosis, lamin meshworks are reassembled around the decondensing chromosomes in daughter cells as the NE forms. Some of the mitotically phosphorylated lamins are recycled for lamin reassembly after dephos-

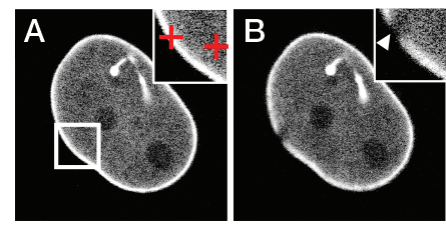

C

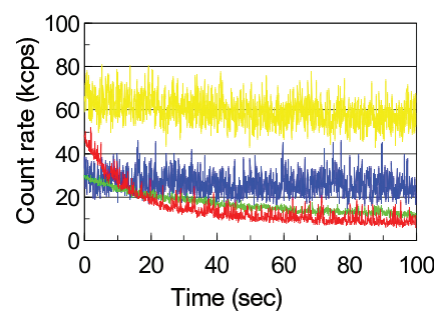

Figure 6. Mobilities of GFP-LA, LB1, LB2, and LC in live HeLa cells measured by FCS. Fluorescence images of GFP-LA before $(A)$ and after $(B)$ FCS measurements. ( $A$, inset) Cross-hairs represent points measured by FCS. ( $B$, inset) Arrowhead indicates the zone within the lamina bleached during FCS measurement. $(C)$ Representative fluorescence fluctuations of GFP-LA, LB1, LB2, and LC in nucleoplasm plotted as a function of time: (yellow line) GFP-LA, (blue line) GFP-LC, (green line) GFP-LB1, (red line) GFP-LB2. (Modified, with permission, from Shimi et al. 2008.) phorylation by type 1 protein phosphatase (PP1) (Thompson et al. 1997). Similar to lamin meshwork disassembly, A-type and B-type lamins are separately reassembled into the assembling lamin meshworks in daughter cells (Moir et al. 2000b). The timing of lamin targeting to the peripheral regions of daughter cell chromosomes varies in different cell types. In mouse keratinocytes (PAM), we found that lamin B1 first accumulates along the peripheries of chromosomes into a relatively stable polymer beginning during the anaphase/telophase transition. Subsequently, lamin A accumulates throughout the nucleoplasm after enclosure of the decondensing chromosomes by the NE in daughter cells (Moir et al. 2000b). In HeLa cells, however, lamin A is localized to the central region of chromosomes close to their spindle attachment sites (designated the "core" region) (Haraguchi et al. 2001). During early telophase, before B-type lamins start assembling around the daughter cell nuclei, lamin A is actively transported into the nucleus at late telophase when the nuclear envelope is formed (Dechat et al. 2004, 2007). FRAP analyses indicate that lamin A is diffusible throughout the nucleoplasm of daughter nuclei during the first few hours of the $\mathrm{G}_{1}$ phase of the cell cycle and becomes stably incorporated into the lamina at later times (Moir et al. 2000b). Lamin reassembly during $\mathrm{G}_{1}$ phase is accompanied by an increase in nuclear size and chromatin decondensation.

We have also studied the role of lamin polymerization during the in vitro assembly of nuclei in Xenopus oocyte extracts. We find that the addition of either the carboxyterminal tail domain or the Ig-fold subdomain of lamin B3 to the Xenopus extracts acts in a dominant negative fashion to prevent lamin polymerization and to inhibit the decondensation of sperm head chromatin and the assembly of the lamina, nuclear membranes, and NPCs comprising the nuclear envelope (Lopez-Soler et al. 2001; Shumaker et al. 2005). These domains also inhibit the polymerization of purified lamin B3 into typical paracrystals in vitro. These results demonstrate that lamin polymerization is required for both chromatin decondensation and NE assembly. Other regulatory mechanisms are also involved in controlling lamin polymerization during the assembly of the lamina. Specifically, we have shown that the nuclear transport factor importin $\alpha$ binds to the nuclear localization sequence (NLS) of lamin B3 in Xenopus interphase oocyte extracts and inhibits lamin assembly (Adam et al. 2008). Similar to the carboxy-terminal and Ig-fold domains, importin $\alpha$ inhibits lamin B3 polymerization into paracrystals in vitro. These results suggest a possible in 
vivo role for importin $\alpha$ in regulating the assembly of the nuclear lamins into the NE-associated lamin meshworks and nucleoplasmic lamin structures.

\section{ROLE OF LAMINS IN CHROMATIN ORGANIZATION}

Our models of nuclear architecture depict lamins and their associated proteins as determinants of chromosome positioning throughout the nucleus (Goldman et al. 2002). Thus, the lamins are considered to be either directly or indirectly involved in anchoring or positioning chromatin at the nuclear lamina, in addition to providing a nucleoplasmic scaffold for organizing chromatin throughout the remaining nucleoplasm. Lamins also appear to have a role in several nuclear "housekeeping" functions that require remodeling of chromatin, including DNA replication and transcription.

\section{NUCLEAR LAMINS: CHROMATIN MODIFICATIONS AND EPIGENETICS}

Interest in the role of nuclear lamins in modifying chromatin and in epigenetics has increased with the findings that a large number of mutations in the human lamin $\mathrm{A}$ gene $(L M N A)$ cause a remarkable number of different diseases, including muscular dystrophies, lipodystrophies, cardiomyopathies, and the premature aging disease Hutchinson-Gilford progeria syndrome (HGPS, or progeria) (Dechat et al. 2008).

Studies of skin fibroblasts obtained from biopsies of HGPS patients have provided new insights into the roles of lamins in regulating chromatin organization. The most common mutation in the nuclear lamin A gene causing HGPS is the single nucleotide substitution, $1824 \mathrm{C}>\mathrm{T}$ (G608G). This substitution causes the formation of an aberrantly spliced mRNA, resulting in the deletion of 50 amino acids in the carboxy-terminal tail domain of lamin A (Eriksson et al. 2003). The deleted amino acids include the second proteolytic cleavage site involved in the normal processing of lamin A, which resides 15 residues upstream of the carboxy-terminal - CAAX box. This mutation results in the synthesis of "progerin," a mutant form of lamin A that is permanently farnesylated and carboxymethylated (Dechat et al. 2007). Progerin expression causes an abnormal thickening of the nuclear lamina, the formation of misshapen or blebbed nuclei, and a dramatic loss of heterochromatin both in the lamina region and throughout the nucleoplasm (Goldman et al. 2004). Cells expressing progerin also exhibit a decrease in the epigenetic mark for facultative heterochromatin, histone $\mathrm{H} 3$ trimethylated on lysine 27 (H3K27me3) (Shumaker et al. 2006). Histone H3 (H3K9me3), a mark for constitutive heterochromatin, also decreases significantly, especially in the pericentric heterochromatin regions of HGPS fibroblasts, suggesting a possible up-regulation in the transcriptional activity of pericentric regions. In support of this, we have found an increase in the expression of sat III RNA from the pericentric region of chromosome 9. We have also observed an increase in the trimethylation of
H4K20 (H4K20me3) in HGPS cells (Shumaker et al. 2006). Concurrent with these epigenetic changes, there is a $90 \%$ decrease in the expression of the H3K27 methyltransferase KMT6 (EZH2). In addition to these changes in histone methylation, HP1 $\alpha$, which is normally associated with $\mathrm{H} 3 \mathrm{~K} 9 \mathrm{me} 3$, appears to be down-regulated and partially dissociated from $\mathrm{H} 3 \mathrm{~K} 9 \mathrm{me} 3$ in HGPS fibroblasts. The expression of progerin also results in the loss of $\mathrm{H} 3 \mathrm{~K} 27 \mathrm{me} 3$ on the inactive X chromosome (Xi) in female HGPS patients, and this is associated with decondensation of the Xi (Shumaker et al. 2006). These changes frequently occur prior to alterations in nuclear shape and do not result in a dissociation of XIST RNA from the Xi. The alterations in histone methylation patterns and chromatin organization that we have detected in the cells derived from progeria patients undoubtedly contribute to their premature aging phenotype. Most likely the changes caused by the lamin A progeria mutations alter normal gene-expression patterns in the various cell and tissue types affected in this devastating disease of children (Shumaker et al. 2006). These studies demonstrate that the interactions of chromatin with lamins, whether direct or indirect, have a profound impact on epigenetic modifications.

Other atypical forms of progeria have provided additional support for the role of lamins in interphase chromosome organization (Taimen et al. 2009). Unlike the most common form of HGPS, patients expressing lamins $\mathrm{A}$ and $\mathrm{C}$ with the point mutation $\mathrm{E} 145 \mathrm{~K}$ in the $1 \mathrm{~B}$ segment of the central rod domain have flower-shaped nuclei with centrally clustered centromeres and abnormal peripherally displaced telomeres (Fig. 7). These changes in chromatin organization suggest that chromosome territories are abnormally arranged during interphase. The abnormal shapes and chromosome configurations seen in the nuclei of E145K lamin A-expressing cells are related to the defective polymerization properties of this mutant protein. This is supported by the finding that E145K lamin A forms disorganized paracrystals in vitro, a defect not seen in the assembly of progerin into higher-order structures (see Fig. 3) (Taimen et al. 2009).
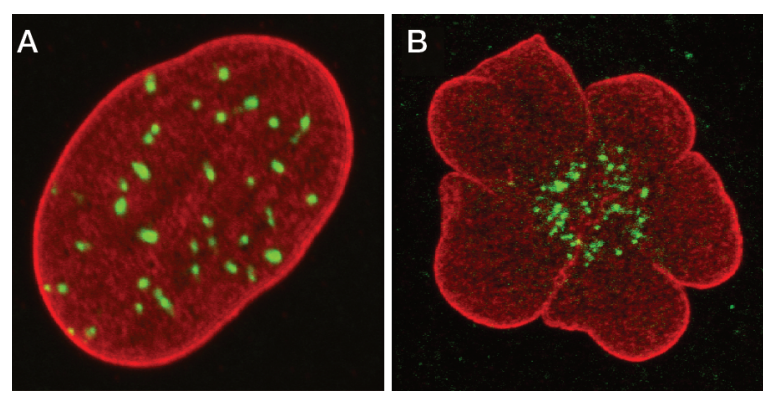

Figure 7. Mislocalization of centromeres in atypical-progeria-patient fibroblasts expressing lamin A E145K. Shown are maximum projections of series of $z$ sections spanning the entire nucleus in cells stained with CREST antiserum (green) and anti-lamin A (red). Centromeres are clustered in the central region of the nucleus in E145K cells $(B)$, whereas they are either closely associated with the peripheral lamina region or elsewhere in the nucleoplasm in control cells $(A)$. (Adapted from Taimen et al. 2009.) 


\section{NUCLEAR LAMINS AND TRANSCRIPTION}

As mentioned above, we have shown that the silencing of lamin B1 expression in HeLa cells induces the formation of NE blebs enriched in lamin $\mathrm{A} / \mathrm{C}$ and devoid of lamin B2. The chromatin within these blebs stains weakly with Hoechst and is enriched in acetylated histones $(\mathrm{AcH} 3)$, suggesting an enrichment for euchromatin (Shimi et al. 2008). In contrast, the presence of centromeric and facultative heterochromatin is greatly decreased within blebs. These and other observations suggest that lamin $\mathrm{A} / \mathrm{C}$ microdomains are associated with gene-rich regions of chromosomes, whereas B-type lamins are involved in organizing gene-poor chromosomal regions and anchoring them to the nuclear lamina (Shimi et al. 2008).

Normal lamin structure and assembly are also required for RNA Pol II transcription. Microinjection of a dominant negative lamin A missing the amino-terminal head domain into BHK 21 cells or the addition of the protein to transcriptionally active cell-free preparations of Xenopus nuclei inhibits RNA Pol II transcription but has no effect on RNA Pol I or Pol III (Spann et al. 2002). Furthermore, the lamin A/C-rich blebs induced by lamin B1 silencing are enriched in the activated hyperphosphorylated form of RNA Pol II (Pol IIo S2 and S5), yet RNA Pol II transcriptional activity is inhibited in these microdomains (Shimi et al. 2008). Several histone marks for activated genes $(\mathrm{AcH} 3$ and H3K4me3) are also present in the blebs, but not H3K36me3, which is normally associated with transcript elongation. This combination of factors suggests that genes in these lamin $\mathrm{A} / \mathrm{C}$ microdomains are in a promoter-proximal stalled state of transcription. To further demonstrate the gene-rich nature of the lamin $\mathrm{A} / \mathrm{C}$-containing blebs, we have isolated blebs by laser microdissection and amplified their DNA for comparative genomic hybridization analyses. The results show an overrepresentation of several gene-rich chromosomal regions in blebs, including the short arm of chromosome 6 (6p) and segments of chromosomes 17 and 19. In addition, the gene-poor chromosome 18 is underrepresented. These results have been confirmed by fluorescence in situ hybridization (FISH) using chromosome-specific probes (Shimi et al. 2008).

On the basis of these studies, we have proposed that Btype lamins, both in the lamina and nucleoplasm, assemble into relatively stable structures that provide a scaffold for gene-poor chromosomal regions and that A-type lamins are preferentially associated with gene-rich chromosomal regions. We also suggest that the interactions between Atype and B-type lamins facilitate cross talk between different nuclear microdomains involved in regulating chromatin organization, positioning, and gene expression.

\section{ROLE OF LAMINS IN DNA REPLICATION}

Several lines of evidence suggest that lamins have a role in regulating DNA replication. Early experiments showed that lamins are associated with DNA replication factories or foci detected by colocalization with newly incorporated bromodeoxyuridine (BrdU) and replication factors such as proliferating cell nuclear antigen (PCNA) (Moir et al. 1994). In Xenopus nuclei assembled in vitro, DNA repli- cation is inhibited following the disruption of endogenous lamin organization by the dominant negative amino-terminally deleted lamin (Spann et al. 1997; Moir et al. 2000a). The addition of this dominant negative mutant also causes the redistribution of endogenous lamins into nuclear foci along with the DNA replication elongation factors PCNA and replication factor C (RFC). In contrast, under these conditions, the DNA replication initiation factors DNA polymerase $\alpha$, minichromosome maintenance protein 3 (MCM3), and the origin recognition complex (ORC) protein Orc2 appear to function normally, as supported by the synthesis of short pieces of DNA that correspond to Okazaki fragments (Spann et al. 1997; Moir et al. 2000a). This inhibitory effect is completely reversed by transferring the lamin-disrupted nuclei into Xenopus interphase extracts devoid of the mutant lamin. Moreover, DNA replication in Xenopus nuclei assembled in vitro is also inhibited by immunodepletion of lamin B3, the major lamin in Xenopus eggs (Lopez-Soler et al. 2001; Shumaker et al. 2005, 2008). The mechanisms responsible for the requirement of normal lamin networks for the chain elongation phase of DNA replication are most likely related to the finding that lamins bind PCNA. Furthermore, the binding site appears to be located within the highly conserved Ig-fold motif in the carboxyl terminus of both the A-type and B-type lamins. This site is known to be important in facilitating protein/protein interactions (Shumaker et al. 2008). Taken together, these results show that lamins are involved in the regulation of PCNA activity in DNA replication and further suggest that they are involved in other processes requiring PCNA, such as DNA repair.

\section{ACKNOWLEDGMENTS}

This work is supportd by the National Cancer Institute, the National Institute on Aging, the Ellison Medical Foundation, and the Progeria Research Foundation.

\section{REFERENCES}

Aaronson RP, Blobel G. 1975. Isolation of nuclear pore complexes in association with a lamina. Proc Natl Acad Sci 72: 1007-1011.

Adam SA, Sengupta K, Goldman RD. 2008. Regulation of nuclear lamin polymerization by importin $\alpha$. J Biol Chem 283: 84628468.

Aebi U, Cohn J, Buhle L, Gerace L. 1986. The nuclear lamina is a meshwork of intermediate-type filaments. Nature 323: 560-564.

Dechat T, Gajewski A, Korbei B, Gerlich D, Daigle N, Haraguchi T, Furukawa K, Ellenberg J, Foisner R. 2004. LAP2 $\alpha$ and BAF transiently localize to telomeres and specific regions on chromatin during nuclear assembly. J Cell Sci 117: 6117-6128.

Dechat T, Shimi T, Adam SA, Rusinol A, Andres DA, Spielmann HP, Sinensky MS, Goldman RD. 2007. Alterations in mitosis and cell cycle progression caused by a mutant lamin A known to accelerate human aging. Proc Natl Acad Sci 104: 4955-4960.

Dechat T, Pfleghaar K, Sengupta K, Shimi S, Shumaker DK, Solimando L, Goldman RD. 2008. Nuclear lamins: Major factors in the structural organization and function of the nucleus and chromatin. Genes Dev 22: 832-853.

Delbarre E, Tramier M, Coppey-Moisan M, Gaillard C, Courvalin JC, Buendia B. 2006. The truncated prelamin A in HutchinsonGilford progeria syndrome alters segregation of A-type and Btype lamin homopolymers. Hum Mol Genet 15: 1113-1122.

Dessev GN, Goldman RD. 1988. Meiotic breakdown of nuclear envelope in oocytes of Spisula solidissima involves phosphory- 
lation and release of nuclear lamin. Dev Biol 130: 543-550.

Dessev GN, Iovcheva C, Tasheva B, Goldman RD. 1988. Protein kinase activity associated with the nuclear lamina. Proc Natl Acad Sci 85: 2994-2998.

Dessev GN, Palazzo R, Rebhun LI, Goldman RD. 1989. Disassembly of the nuclear envelope of Spisula oocytes in a cell free system. Dev Biol 131: 496-504.

Dessev GN, Iovcheva-Dessev C, Goldman RD. 1990. Lamin dimers: Presence in the nuclear lamina of surf clam oocytes and release during nuclear envelope breakdown. J Biol Chem 265: 12636-12641.

Dessev GN, Iovcheva-Dessev C, Bischoff JR, Beach D, Goldman RD. 1991. A complex containing p34cdc2 and cyclin B phosphorylates the nuclear lamin and disassembles nuclei of clam oocytes in vitro. J Cell Biol 112: 523-533.

Eriksson M, Brown WT, Gordon LB, Glynn MW, Singer J, Scott L, Erdos MR, Robbins CM, Moses TY, Berglund P, et al. 2003. Recurrent de novo point mutations in lamin A cause Hutchinson-Gilford progeria syndrome. Nature 423: 293-298.

Fawcett DW. 1966. On the occurrence of a fibrous lamina on the inner aspect of the nuclear envelope in certain cells of vertebrates. Am J Anat 119: 129-145.

Georgatos SD, Pyrpasopoulou A, Theodoropoulos PA. 1997. Nuclear envelope breakdown in mammalian cells involves stepwise lamina disassembly and microtubule-drive deformation of the nuclear membrane. J Cell Sci 110: 2129-2140.

Gerace L, Blum A, Blobel G. 1978. Immunocytochemical localization of the major polypeptides of the nuclear pore complexlamina fraction. Interphase and mitotic distribution. J Cell Biol 79: $546-566$.

Goldman AE, Maul G, Steinert PM, Yang HY, Goldman RD. 1986. Keratin-like proteins that coisolate with intermediate filaments of BHK-21 cells are nuclear lamins. Proc Natl Acad Sci 83: 3839-3843.

Goldman AE, Moir RD, Montag-Lowy M, Stewart M, Goldman RD. 1992. Pathway of incorporation of microinjected lamin A into the nuclear envelope. J Cell Biol 119: 725-735.

Goldman RD, Gruenbaum Y, Moir RD, Shumaker DK, Spann TP. 2002. Nuclear lamins: Building blocks of nuclear architecture. Genes Dev 16: 533-547.

Goldman RD, Shumaker DK, Erdos MR, Eriksson M, Goldman AE, Gordon LB, Gruenbaum Y, Khuon S, Mendez MG, Varga R, Collins FS. 2004. Accumulation of mutant lamin A causes progressive changes in nuclear architecture in Hutchinson-Gilford progeria syndrome. Proc Natl Acad Sci 101: 8963-8968.

Haraguchi T, Koujin T, Segura-Totten M, Lee KK, Matsuoka Y, Yoneda Y, Wilson KL, Hiraoka Y. 2001. BAF is required for emerin assembly into the reforming nuclear envelope. $J$ Cell Sci 114: 4575-4585.

Heald R, McKeon F. 1990. Mutations of phosphorylation sites in lamin A that prevent nuclear lamina disassembly in mitosis. Cell 61: 579-589.

Kandert S, Lüke Y, Kleinhenz T, Neumann S, Lu W, Jaeger VM, Munck M, Wehnert M, Müller CR, Zhou Z, et al. 2007. Nesprin-2 giant safeguards nuclear envelope architecture in LMNA S143F progeria cells. Hum Mol Genet 16: 2944-2959. Erratum. 2008. Hum Mol Genet 17: 468.

Lopez-Soler RI, Moir RD, Spann TP, Stick R, Goldman RD. 2001. A role for nuclear lamins in nuclear envelope assembly. $J$ Cell Biol 154: 61-70.

Ma L, Tsai MY, Wang S, Lu B, Chen R, Iii JR, Zhu X, Zheng Y. 2009. Requirement for Nudel and dynein for assembly of the lamin B spindle matrix. Nat Cell Biol 11: 247-256.
McKeon FD, Kirschner MW, Caput D. 1986. Homologies in both primary and secondary structure between nuclear envelope and intermediate filament proteins. Nature 319: 463-468.

Moir RD, Montag-Lowy M, Goldman RD. 1994. Dynamic properties of nuclear lamins: Lamin B is associated with sites of DNA replication. J Cell Biol 125: 1201-1212.

Moir RD, Spann TP, Herrmann H, Goldman RD. 2000a. Disruption of nuclear lamin organization blocks the elongation phase of DNA replication. J Cell Biol 149: 1179-1192.

Moir RD, Yoon M, Khuon S, Goldman RD. 2000b. Nuclear lamins $\mathrm{A}$ and B1: Different pathways of assembly during nuclear envelope formation in living cells. J Cell Biol 151: 1155-1168.

Parry AD, Conway JF, Goldman AE, Goldman RD, Steinert PM. 1987. Nuclear lamin proteins: Common structures for paracrystalline, filamentous and lattice forms. Int J Biol Macromol 9: $137-145$.

Patrizi G, Poger M. 1967. The ultrastructure of the nuclear periphery. The zonula nucleum limitans. J Ultrastruct Res 17: 127-136.

Peter M, Nakagawa J, Dorée M, Labbé JC, Nigg EA. 1990. In vitro disassembly of the nuclear lamina and $\mathrm{M}$ phase-specific phosphorylation of lamins by cdc2 kinase. Cell 61: 591-602.

Shimi T, Pfleghaar K, Kojima SI, Pack CG, Solovei I, Goldman AE, Adam SA, Shumaker DK, Kinjo M, Cremer T, Goldman RD. 2008. The A- and B-type nuclear lamin networks: Microdomains involved in chromatin organization and transcription. Genes Dev 22: 3409-3421.

Shumaker DK, Lopez-Soler RI, Adam SA, Herrmann H, Moir RD, Spann TP, Goldman RD. 2005. Functions and dysfunctions of the Lamin A Ig-fold domain: Nuclear assembly, growth and Emery-Dreifuss muscular dystrophy. Proc Natl Acad Sci 102: 15494-15499.

Shumaker DK, Dechat T, Kohlmaier A, Adam SA, Bozovsky MR, Erdos MR, Eriksson JE, Goldman AE, Khuon S, Collins FS, et al. 2006. Mutant nuclear lamin A leads to progressive alterations of epigenetic control in premature aging. Proc Natl Acad Sci 103: 8703-8708.

Shumaker DK, Solimando L, Sengupta K, Shimi T, Adam SA, Grunwald A, Strelkov SV, Aebi U, Cardoso MC, Goldman RD. 2008. The highly conserved nuclear lamin Ig-fold binds to PCNA: Its role in DNA replication. J Cell Biol 181: 269-280. Spann TP, Moir RD, Goldman AE, Stick R, Goldman RD. 1997. Disruption of nuclear lamin organization alters the distribution of replication factors and inhibits DNA synthesis. J Cell Biol 136: $1201-1212$.

Spann TP, Goldman AE, Wang C, Huang S, Goldman RD. 2002. Alteration of nuclear lamin organization inhibits RNA polymerase II-dependent transcription. J Cell Biol 156: 603-608.

Taimen P, Pfleghaar K, Shimi T, Möller D, Ben-Harush K, Erdos M, Adam SA, Herrmann H, Medalia O, Collins F, et al. 2009. A progeria mutation reveals functions for lamin A in nuclear assembly, architecture and chromosome organization. Proc Natl Acad Sci 106: 20788-20793.

Thompson LJ, Bollen M, Fields AP. 1997. Identification of protein phosphatase 1 as a mitotic lamin phosphatase. J Biol Chem 272: 29693-29697.

Tsai MY, Wang S, Heidinger JM, Shumaker DK, Adam SA, Goldman RD, Zheng Y. 2006. A mitotic lamin B matrix induced by RanGTP required for spindle assembly. Science 311: 18871893.

Zackroff RV, Goldman AE, Jones JC, Steinert PM, Goldman RD. 1984. Isolation and characterization of keratin-like proteins from cultured cells with fibroblastic morphology. J Cell Biol 98: $1231-1237$. 


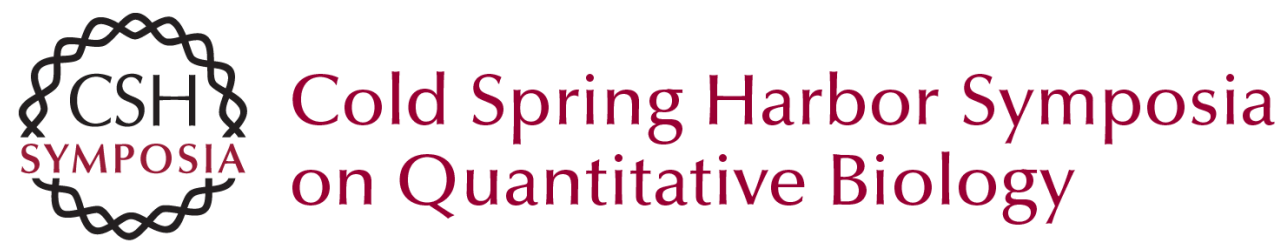

\section{Nuclear Lamins in Cell Regulation and Disease}

T. Shimi, V. Butin-Israeli, S.A. Adam, et al.

Cold Spring Harb Symp Quant Biol 2010 75: 525-531 originally published online April 5, 2011

Access the most recent version at doi:10.1101/sqb.2010.75.045

References This article cites 42 articles, 28 of which can be accessed free at:

http://symposium.cshlp.org/content/75/525.full.html\#ref-list-1

License

Email Alerting Receive free email alerts when new articles cite this article - sign up in the box at the Service top right corner of the article or click here.

To subscribe to Cold Spring Harbor Symposia on Quantitative Biology go to:

http://symposium.cshlp.org/subscriptions 